\title{
CIENCIAMATRIA
}

Revista Interdisciplinaria de Humanidades, Educación, Ciencia y Tecnología

Año VIII. Vol. VIII. Nro 1. Edición Especial. 2022

Hecho el depósito de ley: pp201602FA4721

ISSN-L: 2542-3029; ISSN: 2610-802X

Universidad Nacional Experimental Francisco de Miranda (UNEFM). Santa Ana de Coro. Venezuela

Alexandra-del-Rocío Maldonado-Núñez; Zila Isabel Esteves-Fajardo

DOI 10.35381/cm.v8i1.656

\section{Habilidades del docente investigador en Educación Superior}

Teacher's research skills in higher education

\author{
Alexandra-del-Rocío Maldonado-Núñez \\ alexandra.maldonadon@ug.edu.ec \\ Universidad de Guayaquil. Guayaquil \\ Ecuador \\ https://orcid.org/0000-0002-6532-4300 \\ Zila Isabel Esteves-Fajardo \\ zila.estevesf@ug.edu.ec \\ Universidad de Guayaquil, Guayaquil \\ Ecuador \\ https://orcid.org/0000-0002-2283-5370
}

Recibido: 15 de septiembre 2021

Revisado: 10 de noviembre 2021

Aprobado: 15 de diciembre 2021

Publicado: 01 de enero de 2022 
CIENCIAMATRIA

Revista Interdisciplinaria de Humanidades, Educación, Ciencia y Tecnología

Año VIII. Vol. VIII. Nro 1. Edición Especial. 2022

Hecho el depósito de ley: pp201602FA4721

ISSN-L: 2542-3029; ISSN: 2610-802X

Universidad Nacional Experimental Francisco de Miranda (UNEFM). Santa Ana de Coro. Venezuela

Alexandra-del-Rocío Maldonado-Núñez; Zila Isabel Esteves-Fajardo

\title{
RESUMEN
}

El presente artículo devela un análisis documental acerca de las habilidades que los docentes deben poseer al momento de realizar investigaciones. El estudio tuvo como propósito analizar las habilidades investigativas del docente universitario al momento de indagar a profundidad el contexto educativo en el cual se desenvuelve. Se empleó la metodología documental, a fin de reflexionar sobre la importancia que tiene la investigación dentro del entorno de la educación universitaria. Como resultado, la investigación constituyó un elemento clave para los docentes de todas las áreas, ya que permitió comprender cómo se pueden alcanzar las metas que contribuyen al mejoramiento y progreso del proceso educativo en los diversos campos del saber. Asimismo, se pudieron definir los distintos rasgos apropiados que el docente debe considerar para el quehacer investigativo, tales como: analítico de su propio contexto educativo, constructor racional de la realidad, innovador, transformador y promotor activo, garante de una educación óptima.

Descriptores: Habilidad; educación superior; investigación. (Palabras tomadas de Tesauro Unesco).

\begin{abstract}
This article reveals a documentary analysis about the skills that teachers should have when conducting research. The purpose of the study was to analyze the investigative skills that college professors own when investigating in depth the educational context in which they operate. The documentary methodology was used in order to reflect on the importance of research within the environment of university education. As a result, research is a key element for professors in all areas, since it allowed comprehending how goals might be achieved for contributing to the improvement and progress of the educational process in the various fields of knowledge. Likewise, it was possible to define the different appropriate traits that the teachers should consider for the investigative task, such as: analytical of their own educational context, rational constructor of reality, innovative, transformer and active promoter, guarantor of an optimal and quality education.
\end{abstract}

Descriptors: Skill; higher education; research. (Words taken from Unesco Thesaurus). 


\section{CIENCIAMATRIA \\ Revista Interdisciplinaria de Humanidades, Educación, Ciencia y Tecnología \\ Año VIII. Vol. VIII. Nro 1. Edición Especial. 2022 \\ Hecho el depósito de ley: pp201602FA4721 \\ ISSN-L: 2542-3029; ISSN: 2610-802X \\ Universidad Nacional Experimental Francisco de Miranda (UNEFM). Santa Ana de Coro. Venezuela}

Alexandra-del-Rocío Maldonado-Núñez; Zila Isabel Esteves-Fajardo

\section{INTRODUCCIÓN}

Hoy en día, el docente universitario enfrenta una serie de retos que lo conducen no sólo a dar continuidad a su formación sino a desarrollar capacidades que le permiten indagar y comprender su entorno para contribuir a mejorarlo. En tal sentido, es menester que el mismo se comprometa con su contexto educativo y, para ello, es necesario acudir a la indagación profunda de su entorno laboral como ente activo, lo cual implica interesarse por el progreso del hecho educativo y del aprendizaje apropiado de los estudiantes como protagonistas de la acción.

Al respecto, vale resaltar lo expuesto por (Reiban, 2018, p 1), quien manifiesta lo siguiente: "La universidad precisa docentes con elevados niveles de competencia investigativa y con una cultura de la ciencia que le facilite ejecutar procesos de investigación como lo precisa la sociedad actual".

En atención a tal apreciación, se hace necesario que el profesor universitario se prepare para asumir diversos retos que lo conduzcan a reflexionar sobre su labor, el desempeño estudiantil y sobre las alternativas posibles para llevar a cabo un proceso educativo óptimo y de calidad.

Este artículo tiene sus bases en este tema de interés para el entorno educativo universitario, ya que a través de la investigación se logran alcanzar diversas metas en beneficio para la comunidad que hace vida tanto dentro como fuera de un centro universitario. Ahora bien, ¿de qué manera contribuye la habilidad investigativa en el proceso educativo universitario? Para dar respuesta a esta interrogante, vale citar diversos autores que, de una u otra forma, aportan sus ideas para el alcance de una instrucción efectiva.

Desde el punto de vista del pensar, (Delgado y Alfonzo, 2019) exponen que dicho pensar, desde la acción investigativa, sucede a partir de su propio desempeño didáctico, lo cual implica ser consciente de la realidad que se vivencia a diario en función de lo reflexivo. En este sentido, el docente debe estar constantemente razonando sobre su desempeño, 


\section{CIENCIAMATRIA \\ Revista Interdisciplinaria de Humanidades, Educación, Ciencia y Tecnología \\ Año VIII. Vol. VIII. Nro 1. Edición Especial. 2022 \\ Hecho el depósito de ley: pp201602FA4721 \\ ISSN-L: 2542-3029; ISSN: 2610-802X \\ Universidad Nacional Experimental Francisco de Miranda (UNEFM). Santa Ana de Coro. Venezuela}

Alexandra-del-Rocío Maldonado-Núñez; Zila Isabel Esteves-Fajardo

reconsiderando aquellas funciones que son adecuadas y desechando las no apropiadas, con el propósito de cooperar con un sistema educativo eficaz para el aprendizaje de sus estudiantes.

Por otro lado, desde la visión de competencias (Ollarves y Salguero, 2009, p. 122) las definen como aquellas constituidas por "un componente individual que se demuestra y componente organizacional que se manifiesta a través de la productividad, calidad o innovación". Por consiguiente, las habilidades investigativas se figuran como aquellas capacidades que el docente adquiere para crear y generar nuevos conocimientos, a objeto de promover la inventiva, el talento y la motivación hacia el fomento de novedosas ideas que despierten en el estudiante su interés hacia la inquisición.

De igual modo, tomando como referencia la perspectiva representativa de las habilidades investigativas (Ollarves y Salguero, 2009), mencionan como puntos relevantes: el establecimiento de políticas y metas, experiencias propias de los individuos, líneas de investigación y cultura investigativa de impacto social. Todos estos aspectos se asumen en el presente artículo como bases fundamentales, por cuanto mediante ellos se logra llevar a cabo diversos estudios científicos organizados contentivos de sustentos teóricos válidos y sustentados en la realidad de cada individuo, con el fin de no sólo proponer metas sino también alcanzarlas a través del accionar de los propios protagonistas del contexto a investigar.

Para este estudio, se planteó como objetivo general: analizar las habilidades investigativas del docente universitario. También se constituyeron como propósitos específicos: realizar una revisión teórica sobre las habilidades investigativas del docente universitario y efectuar un análisis reflexivo que conduzca al establecimiento de una visión global sobre el eficiente rol del docente como investigador.

Tomando como base lo anteriormente expuesto, vale resaltar la importancia del presente escrito, puesto que supone un artículo de orientación para todo el contexto educativo, 


\section{CIENCIAMATRIA}

Revista Interdisciplinaria de Humanidades, Educación, Ciencia y Tecnología

Año VIII. Vol. VIII. Nro 1. Edición Especial. 2022

Hecho el depósito de ley: pp201602FA4721

ISSN-L: 2542-3029; ISSN: 2610-802X

Universidad Nacional Experimental Francisco de Miranda (UNEFM). Santa Ana de Coro. Venezuela

Alexandra-del-Rocío Maldonado-Núñez; Zila Isabel Esteves-Fajardo

describiendo el acontecer investigativo que se vivencia en el nivel universitario y el impacto social que esto tiene sobre la sociedad.

\section{Referencial teórico}

\section{Desarrollo de habilidades investigativas}

Poveda y Chirino, (2015) manifiestan que la sociedad actual demanda una serie de cambios constantes que requieren de atención y hacen que se asuman nuevos retos a objeto de favorecer el proceso de enseñanza y aprendizaje dando paso a la invención y a lo novedoso. En relación con estos autores, vale decir que se corrobora la importancia de la investigación como eje esencial del ser humano, puesto que hoy en día son muchas las evoluciones de las que somos partícipes y requieren ser tratadas para lograr ajustarnos ellas.

Tomando como referencia lo antes mencionado, cabe preguntarse ¿Cómo desarrollar habilidades investigativas en el acontecer educativo universitario? Parece difícil pero no lo es, a continuación se precisan varias recomendaciones que podrían contribuir con el hacer científico dentro del contexto universitario:

-Ser activo, un docente activo es aquel que siempre está comprometido con su proceso de enseñanza, indaga acerca de las mejores alternativas para mejorar tanto su propio desempeño como el de sus estudiantes e inclusive el de la institución donde labora.

- Ser responsable, hace referencia a aquellos docentes que nunca olvidan que su labor requiere del cumplimiento cabal de sus funciones, por ende la responsabilidad constituye un valor fundamental para el desarrollo de diversas investigaciones al día y en pro del mejoramiento de los problemas del aquí y el ahora.

-Ser integral, un docente busca crecer a nivel profesional y también el avance del aprendizaje de sus estudiantes. Asimismo, labora en función del beneficio de la comunidad y se involucra con otros profesionales para trabajar en equipo y ejecutar proyectos multidisciplinarios en pro del bien común. 


\section{CIENCIAMATRIA \\ Revista Interdisciplinaria de Humanidades, Educación, Ciencia y Tecnología \\ Año VIII. Vol. VIII. Nro 1. Edición Especial. 2022 \\ Hecho el depósito de ley: pp201602FA4721 \\ ISSN-L: 2542-3029; ISSN: 2610-802X \\ Universidad Nacional Experimental Francisco de Miranda (UNEFM). Santa Ana de Coro. Venezuela}

Alexandra-del-Rocío Maldonado-Núñez; Zila Isabel Esteves-Fajardo

-Ser reflexivo, un docente debe tener presente la autoevaluación de su desempeño, por cuanto esto le permite reconocer sus debilidades y fortalezas, a fin de mejorar su acción pedagógica y andragógica.

En términos generales, el desarrollo de las habilidades investigativas constituye la unión de diversos elementos como: su desempeño activo, su valor hacia la responsabilidad, su autorreflexión, entre otros aspectos, que contribuyen a la construcción de un proceso educativo universitario óptimo.

\section{Rasgos del contexto universitario para la producción investigativa}

El entorno universitario engloba una serie de elementos que consolidan la institución y la hacen dinámica dentro de la sociedad. De esta forma, se destaca el rol de la formación, aludiendo a diversas disciplinas:

Por ejemplo, en el caso de disciplinas como la geología o física pertenecientes a las ciencias naturales, formación pudiera aludir a la formación natural-externa de las cosas como las formaciones rocosas, formaciones coloidales, formaciones de masas de aire. En el caso de disciplinas como la filosofía, psicología, pedagogía, pertenecientes a las ciencias humanas, el uso del término formación tiende a apuntar a las características sociales-internas del sujeto, formación de sujetos críticos, pensantes, educativos. (Navarrete, 2013, p. 7)

Considerando lo expuesto por Navarrete, es necesario responder a lo siguiente: ¿De qué manera se puede abordar cada disciplina mediante la investigación? Como se puede evidenciar, la formación en diversos campos amerita de la investigación, ya que cada uno constituye un área distinta con sus propias particularidades, las cuales requieren ser exploradas a profundidad para ser comprendidas, tratadas y mejoradas si las mismas así lo requieren.

En el caso de la filosofía, la cual incluye la epistemología, el campo científico se aborda teniendo presente "el diseño de la investigación y las consideraciones éticas que deben envolver al trabajo investigativo" (Rivas, 2011, p. 36). Esta aseveración permite 


\section{CIENCIAMATRIA \\ Revista Interdisciplinaria de Humanidades, Educación, Ciencia y Tecnología \\ Año VIII. Vol. VIII. Nro 1. Edición Especial. 2022 \\ Hecho el depósito de ley: pp201602FA4721 \\ ISSN-L: 2542-3029; ISSN: 2610-802X \\ Universidad Nacional Experimental Francisco de Miranda (UNEFM). Santa Ana de Coro. Venezuela}

Alexandra-del-Rocío Maldonado-Núñez; Zila Isabel Esteves-Fajardo

determinar que la investigación va más allá de la indagación, puesto que incluye la consideración de los valores como elementos esenciales de una investigación eficaz.

Por su parte, (Tornimbeni, González, Corigliani, y Co. 2011) señalan una serie de dimensiones que un estudiante de psicología debe considerar para aprender investigación, entre las cuales destacan además, opiniones de entrevistas que los autores hicieron; estas son: conocimiento y dominio personal enfocado en la autorregulación, la curiosidad y la actitud crítica. También mencionan la gestión de relaciones abarcando el trabajo en equipo y la colaboración. Finalmente, en la dimensión cognitiva destacan el pensamiento analítico, la experticia profesional y el pensamiento sistémico.

Desde el plano pedagógico y de acuerdo con (Estrada, 2014), la adquisición de la habilidad investigativa en el plano educativo formal es considerada durante todo el proceso académico; por ende, asevera lo siguiente:

La formación y desarrollo de la competencia investigativa ha sido concebida, en la educación superior, tanto en el plano procesal-práctico y estructuralformal del currículo a lo largo de la carrera universitaria, aunque tiene su máxima expresión en la realización de tesis de pregrado y trabajos investigativos finales de diversas asignaturas, ya que el estudiante debe evidenciar el uso de la metodología de la investigación científica en solución de un problema investigativo aplicando para ello todas las etapas de la investigación científica.

Sin embargo, la investigación siempre está presente aún en los momentos cuando se asignan tareas sencillas al estudiante para indagar sobre conocimientos nuevos, puesto que mínima idea novedosa puede dar lugar a la ejecución de un proyecto innovador. En este particular, vale señalar que el docente como promotor de la investigación, puede tener la habilidad de trabajar en equipo con sus educandos para fomentar la acción investigativa en el devenir de una carrera universitaria. 


\section{CIENCIAMATRIA \\ Revista Interdisciplinaria de Humanidades, Educación, Ciencia y Tecnología \\ Año VIII. Vol. VIII. Nro 1. Edición Especial. 2022 \\ Hecho el depósito de ley: pp201602FA4721 \\ ISSN-L: 2542-3029; ISSN: 2610-802X \\ Universidad Nacional Experimental Francisco de Miranda (UNEFM). Santa Ana de Coro. Venezuela}

Alexandra-del-Rocío Maldonado-Núñez; Zila Isabel Esteves-Fajardo

Todos estos aspectos confirman todas aquellas competencias y cualidades que deben considerar los docentes investigadores no únicamente en el campo de la psicología sino en otros, ya que coincide con la manera de actuar de un investigador en el ámbito social, económico, político, educativo, etcétera.

Para resumir lo antes dicho, vale resaltar que los rasgos que debe reunir el contexto universitario para la producción investigativa tomando en cuenta los autores citados, se sustentan en los valores, la autorregulación, la curiosidad, la actitud crítica, el trabajo en equipo y la colaboración. Todo ello aplica para los distintos campos del saber como: la psicología, la filosofía, la física, la química, la educación y otros.

Una vez citados los autores que definen los referentes teóricos de la docencia investigativa universitaria, se procede a especificar la metodología empleada para el desarrollo del presente estudio.

\section{METODOLOGÍA}

La investigación fue de análisis documental, ya que se detallan las características del docente investigador y se hace una reflexión de tales cualidades. En este sentido, Hernández, Fernández y Baptista (2003: 119), señalan que los estudios documentales se fundamentan en la indagación profunda de diversas referencias teóricas. Por ende, se especifican los siguientes rasgos que definen un docente enfocado en la investigación: analítico de su propio contexto educativo, constructor racional de la realidad, innovador, transformador y promotor activo garante de una educación óptima y de calidad; aspectos que serán abordados, a fin de conformar una idea precisa de lo que un docente debe tener presente al momento de asumir su rol de investigador.

Para ahondar un poco más sobre estos temas, se procede a hacer una reflexión sobre las habilidades que un docente investigador debe tomar en cuenta a lo largo de su desempeño laboral. 
CIENCIAMATRIA

Revista Interdisciplinaria de Humanidades, Educación, Ciencia y Tecnología

Año VIII. Vol. VIII. Nro 1. Edición Especial. 2022

Hecho el depósito de ley: pp201602FA4721

ISSN-L: 2542-3029; ISSN: 2610-802X

Universidad Nacional Experimental Francisco de Miranda (UNEFM). Santa Ana de Coro. Venezuela

Alexandra-del-Rocío Maldonado-Núñez; Zila Isabel Esteves-Fajardo

\section{RESULTADOS}

Debido a que la investigación está basada en una revisión teórica y en el análisis de la realidad, los resultados serán presentados en cuadros donde se precisan las diversas particularidades que un formador e investigador debe considerar teniendo en cuenta un enfoque reflexivo de su rol.

\section{Una visión reflexiva del docente investigador en el entorno universitario}

La investigación constituye un valor apreciable a lo largo del quehacer social, por cuanto contribuye a la indagación, el descubrimiento y a la invención de nuevas perspectivas. Por tal razón, es digna de ser descrita y analizada, tomando en cuenta diversas áreas y las experiencias vividas por los actores del proceso.

A este respecto, es menester responder la siguiente incógnita: ¿qué cualidades esenciales debe poseer un docente investigador a nivel universitario? En las siguientes tablas se explican algunos de los rasgos principales que podrían servir de base al desempeño de un buen rol:

\section{Tabla 1.}

Rasgo1. Analítico de su propio contexto educativo.

\begin{tabular}{|l|l|}
\hline \multicolumn{1}{|c|}{ Rasgo } & \multicolumn{1}{|c|}{ Concepción } \\
\hline $\begin{array}{l}\text { Analítico de su propio } \\
\text { contexto educativo. }\end{array}$ & $\begin{array}{l}\text { (Villalobos y de Cabrera, 2009, p. 141) manifiestan que } \\
\text { "asumir una actitud reflexiva contribuye a que los docentes } \\
\text { reconozcan comportamientos y prácticas que inhiben su } \\
\text { potencial para la tolerancia y la aceptación, elementos vitales }\end{array}$ \\
\hline
\end{tabular}




\section{CIENCIAMATRIA}

Revista Interdisciplinaria de Humanidades, Educación, Ciencia y Tecnología

Año VIII. Vol. VIII. Nro 1. Edición Especial. 2022

Hecho el depósito de ley: pp201602FA4721

ISSN-L: 2542-3029; ISSN: 2610-802X

Universidad Nacional Experimental Francisco de Miranda (UNEFM). Santa Ana de Coro. Venezuela

Alexandra-del-Rocío Maldonado-Núñez; Zila Isabel Esteves-Fajardo

\begin{tabular}{|l|l|}
\hline para enfrentar las diferencias de todos los estudiantes en una \\
sociedad heterogénea que se mueve hacia una comunidad \\
donde la globalización es el común denominador".
\end{tabular}

Elaboración: Los autores sustentados en Villalobos y de Cabrera (2009).

De este modo, tales diferencias constituyen la riqueza de la humanidad y, por ello, derivan investigaciones diversas para atender a variedad de situaciones que ameritan ser exploradas y mejoradas en ocasiones, a raíz de la reflexión crítica sobre las mismas. De ahí que la investigación juega un rol fundamental para la solución de los problemas de cada persona o entorno, a fin de buscar las mejores estrategias para el diálogo, la dialéctica y el entendimiento.

\section{Tabla 2.}

Rasgo 2. Constructor racional de la realidad.

\begin{tabular}{|l|l|}
\hline \multicolumn{1}{|c|}{ Rasgo } & \multicolumn{1}{|c|}{ Concepción } \\
\hline Constructor racional la realidad & $\begin{array}{l}\text { Según (Carmona, 2008, p. 134). "El enfoque pedagógico } \\
\text { crítico, reflexivo y creativo, presenta un énfasis en el desarrollo } \\
\text { integral de la persona, de un tipo de ciudadano capaz de tomar } \\
\text { en cuenta el punto de vista de otros, argumentar sus } \\
\text { propuestas y sustentar sus decisiones de manera reflexiva y } \\
\text { creativa, más cooperativo en la solución conjunta de los } \\
\text { problemas y de mayor consistencia moral en su quehacer } \\
\text { cotidiano". }\end{array}$ \\
\hline
\end{tabular}

Elaboración: Los autores sustentados en Carmona (2008). 


\section{CIENCIAMATRIA \\ Revista Interdisciplinaria de Humanidades, Educación, Ciencia y Tecnología \\ Año VIII. Vol. VIII. Nro 1. Edición Especial. 2022 \\ Hecho el depósito de ley: pp201602FA4721 \\ ISSN-L: 2542-3029; ISSN: 2610-802X \\ Universidad Nacional Experimental Francisco de Miranda (UNEFM). Santa Ana de Coro. Venezuela}

Alexandra-del-Rocío Maldonado-Núñez; Zila Isabel Esteves-Fajardo

A este respecto, el docente asume un rol racional en su entorno, por cuanto toma en cuenta las diversas realidades que se presentan en el contexto formativo y se hace parte de un proceso, en el cual, integra a sus estudiantes para lograr la ejecución de diversos proyectos con base teórica consolidada y en pro de buscar alternativas de solución a los problemas del día a día.

\section{Tabla 3.}

Rasgo 3. Innovador.

\begin{tabular}{|l|l|}
\hline \multicolumn{1}{|c|}{ Rasgo } & \multicolumn{1}{|c|}{ Concepción } \\
\hline Innovador & $\begin{array}{l}\text { De acuerdo con (Ríos, 2004, p. 95), autores como (Stenhouse, } \\
\text { 1991; Ghani, 1992; Escudero, 1999, Sansano et al., 1993; } \\
\text { Marcelo, 1996; Ríos, 2003a } \text { "plantean que la acción } \\
\text { innovadora de los profesores, ligada fuertemente a su } \\
\text { capacidad de autonomía, puede permitirles desarrollar un } \\
\text { mejor y mayor rol profesional, lo cual los habilita para ser } \\
\text { capaces de poseer en plenitud una competencia en su área de } \\
\text { conocimiento para diagnosticar situaciones y proponer } \\
\text { diversas alternativas de acción, tomando en consideración los } \\
\text { objetivos a alcanzar y las condiciones específicas donde } \\
\text { realiza su acción docente". }\end{array}$ \\
\hline
\end{tabular}

Elaboración: Los autores sustentados en Ríos (2004).

De este modo, lo expuesto por estos autores reafirma la relevancia de un docente innovador, puesto que al tener esta cualidad, será capaz de motivar al estudiante a producir nuevos conocimientos y a desarrollar estudios en pro del beneficio educativo y social en atención a la consecución de metas distintas. También, tomará en cuenta la 


\section{CIENCIAMATRIA}

Revista Interdisciplinaria de Humanidades, Educación, Ciencia y Tecnología

Año VIII. Vol. VIII. Nro 1. Edición Especial. 2022

Hecho el depósito de ley: pp201602FA4721

ISSN-L: 2542-3029; ISSN: 2610-802X

Universidad Nacional Experimental Francisco de Miranda (UNEFM). Santa Ana de Coro. Venezuela

Alexandra-del-Rocío Maldonado-Núñez; Zila Isabel Esteves-Fajardo

estructuración coherente investigativa por medio de un diagnóstico que conduzca a propuestas de solución y a propuestas de transformación.

\section{Tabla 4.}

Rasgo 4. Transformador.

\begin{tabular}{|l|l|l|}
\hline \multicolumn{1}{|c|}{ Rasgo } & \multicolumn{3}{|c|}{ Concepción } \\
\hline Transformador & $\begin{array}{l}\mid \\
\text { Para (Meza y Flores, 2014, p. 103). "El liderazgo } \\
\text { transformacional, da sentido a lo que se hace e inspira a las } \\
\text { personas a trascender sus propios } \\
\text { intereses por un bien mayor". }\end{array}$ \\
\hline
\end{tabular}

Elaboración: Los autores, sustentado en Meza y Flores (2014).

La idea de un docente investigador es lograr lo expuesto por las autoras mencionadas, es decir, dar lugar a una formación sustentada en la ampliación del saber por medio de diversos métodos, técnicas y estrategias para la generación de novedosos conocimientos fundamentados en la realidad de cada persona.

\section{Tabla 5.}

Rasgo 5. Promotor activo

\begin{tabular}{|l|l|}
\hline \multicolumn{1}{|c|}{ Rasgo } & \multicolumn{1}{c|}{ Concepción } \\
\hline Promotor activo & $\begin{array}{l}\text { (Villalobos y de Cabrera, 2009, p. 156) establecen que " a } \\
\text { medida que los docentes desarrollan la capacidad de ser } \\
\text { auto-reflexivos, se hacen más y más conscientes de cómo son } \\
\text { participantes interactivos de los encuentros constantes en el }\end{array}$ \\
\hline
\end{tabular}


CIENCIAMATRIA

Revista Interdisciplinaria de Humanidades, Educación, Ciencia y Tecnología

Año VIII. Vol. VIII. Nro 1. Edición Especial. 2022

Hecho el depósito de ley: pp201602FA4721

ISSN-L: 2542-3029; ISSN: 2610-802X

Universidad Nacional Experimental Francisco de Miranda (UNEFM). Santa Ana de Coro. Venezuela

Alexandra-del-Rocío Maldonado-Núñez; Zila Isabel Esteves-Fajardo

\begin{tabular}{|l|l|}
\hline & $\begin{array}{l}\text { aula de clases, en lugar de inocentes observadores } 0 \\
\text { víctimas". }\end{array}$ \\
\hline
\end{tabular}

Elaboración: Los autores sustentados en Villalobos y de Cabrera (2009).

Como se puede evidenciar, la autorreflexión va de la mano con el rol de promotor activo, ya que a partir de este pensar, se desencadenan diversas acciones que hacen del docente un ente dinámico olvidando la transmisión de conocimientos para convertirlas en interacción y compartir de saberes. Esto constituye otra razón investigativa, por cuanto es a través de la socialización horizontal que tanto docentes como estudiantes descubren nuevas perspectivas a indagar.

Tabla 6.

Rasgo 6. Garante de una educación óptima y de calidad.

\begin{tabular}{|c|c|}
\hline Rasgos & Concepción \\
\hline $\begin{array}{lll}\text { Garante de una } \\
\text { educación óptima y } \\
\text { de calidad }\end{array}$ & $\begin{array}{l}\text { Para (Hernández, 2009, p. 5), "la investigación se constituye } \\
\text { en una de las actividades primordiales para el } \\
\text { perfeccionamiento de la docencia; ella establece la } \\
\text { interrelación en el quehacer del proceso enseñanza- } \\
\text { aprendizaje". }\end{array}$ \\
\hline
\end{tabular}

Elaboración: Los autores sustentados en Hernández (2009).

Para lograr lo afirmado por la autora, se requiere de un docente cuyo interés gire en torno al alcance de una educación óptima y de calidad, por cuanto si su intención es contribuir al progreso pedagógico y andragógico, se mantendrá motivado y motivará a sus estudiantes a alcanzar sus metas y a solucionar diversas de problemas que se presenten a lo largo de su formación educativa. 


\section{CIENCIAMATRIA \\ Revista Interdisciplinaria de Humanidades, Educación, Ciencia y Tecnología \\ Año VIII. Vol. VIII. Nro 1. Edición Especial. 2022 \\ Hecho el depósito de ley: pp201602FA4721 \\ ISSN-L: 2542-3029; ISSN: 2610-802X \\ Universidad Nacional Experimental Francisco de Miranda (UNEFM). Santa Ana de Coro. Venezuela}

Alexandra-del-Rocío Maldonado-Núñez; Zila Isabel Esteves-Fajardo

Una vez descritos todos los rasgos, se pudo evidenciar que un docente investigador es un ser integral que se preocupa tanto por el bienestar de sus estudiantes como por el suyo propio y de la comunidad que les rodea, lo cual lo conduce a trabajar en equipo con los aprendices para ofrecer alternativas de solución a las distintas situaciones del día a día.

Luego de describir los resultados obtenidos a partir de la revisión teórica, se procede a exponer el apartado de la discusión, donde se hace una breve argumentación del tema en estudio.

\section{DISCUSIÓN}

Tal como se expuso anteriormente, (Hernández, 2009) establece que la investigación involucra mejoramiento del rol docente y la correlación del binomio enseñanzaaprendizaje. Su postura resume lo que engloba el campo investigativo, ya que los diversos estudios surgen del entorno pedagógico y andragógico, los cuales se asumen como aspectos centrales para desarrollar novedosos descubrimientos propicios para describir, analizar, experimentar y transformar una determinada realidad. De ahí la deriva importancia de la habilidad docente, por cuanto es él un modelo a seguir para motivar a la pesquisa de nuevos conocimientos.

Para concluir, a continuación se presentan los aspectos que resumen lo esencial de las habilidades del docente investigador a partir de las perspectivas consultadas.

\section{CONCLUSIONES}

Las habilidades del docente investigador en Educación Superior suponen una serie de cualidades que el mismo debe poseer para motivar a sus estudiantes a la investigación. Todo ello, implica poseer diversos rasgos que despierten en el individuo el interés hacia la exploración de diversos contextos cuyas circunstancias requieren de atención, tratamiento, análisis, transformación e innovación. Todos los autores citados 
contribuyeron a definir algunas de las cualidades fundamentales que debe tener un docente para la investigación. En términos generales, el mismo debe ser analítico de su propio contexto educativo, constructor racional de la realidad, innovador, transformador, promotor activo y garante de una educación óptima y de calidad.

Lo expuesto es simplemente un abrebocas y una invitación a los docentes, a los estudiantes universitarios e inclusive a la comunidad en su totalidad para motivarlos a la ejecución de otras investigaciones que podrían profundizar aún más este tema desde diversas áreas del saber.

\section{FINANCIAMIENTO}

No monetario.

\section{AGRADECIMIENTO}

A todos los agentes sociales que motivaron el desarrollo de la investigación.

\section{REFERENCIAS CONSULTADAS}

Carmona, M. (2008). Hacia una formación docente reflexiva y crítica: fundamentos filosóficos. [Towards a reflective and critical teacher training: philosophical foundations]. Revista de Teoría y Didáctica de las Ciencias Sociales, núm. 13, enero-diciembre, pp. 125146. Universidad de los Andes. Mérida, Venezuela. Recuperado de: https://n9.cl/1os0i

Hernández, I. (2009). El docente investigador en la formación de profesionales. [The research professor in the training of professionals]. Revista Virtual Universidad Católica del Norte, núm. 27, mayo-agosto, pp. 1-21. Fundación Universitaria Católica del Norte Medellín, Colombia. Recuperado de: https://n9.cl/zdpq1

Delgado, Y., Alfonzo, R. (2019) Competencias Investigativas del Docente Construidas durante la Formación Universitaria [Teaching Research Competences built during University Training]. Revista Scientific, vol. 4, núm. 13, pp. 200-220. Recuperado de: https://n9.cl/4tbwb 


\section{CIENCIAMATRIA}

Revista Interdisciplinaria de Humanidades, Educación, Ciencia y Tecnología

Año VIII. Vol. VIII. Nro 1. Edición Especial. 2022

Hecho el depósito de ley: pp201602FA4721

ISSN-L: 2542-3029; ISSN: 2610-802X

Universidad Nacional Experimental Francisco de Miranda (UNEFM). Santa Ana de Coro. Venezuela

Alexandra-del-Rocío Maldonado-Núñez; Zila Isabel Esteves-Fajardo

Estrada Molina, Odiel. (2014). Sistematización teórica sobre la competencia investigativa. [Theoretical systematization on investigative competence]. Revista Electrónica Educare, vol. 18, núm. 2, mayo-, pp. 177-194. Universidad Nacional Heredia, Costa Rica. Recuperado de: https://n9.cl/65w7k

Hernández, R., Fernández, C. y Baptista M. (2015). Metodología de la investigación. [Investigation Metodology]. México: Mc Graw Hill.

Meza, M., Flores, I. (2014. El liderazgo transformacional en el trabajo docente: Colegio Mier y Pesado, un estudio de caso. [Transformational leadership in teaching work: Mier y Pesado School, a case study]. Educación, vol. 38, núm. 1, enero-junio, , pp. 101-115. Universidad de Costa Rica. San Pedro, Montes de Oca, Costa Rica. Recuperado de: https://n9.cl/oxw0k

Navarrete, Z. (2013). La universidad como espacio de Formación profesional y constructora de identidades. [The university as a space for professional training and construction of identities]. Universidades, núm. 57, julio-septiembre, 2013, pp. 5-16 Unión de Universidades de América Latina y el Caribe. Distrito Federal, Organismo Internacional. Recuperado de: https://n9.cl/wn9w4

Ollarves, Y., Salguero, L. (2009) Una propuesta de competencias investigativas para los docentes universitarios. [A proposal of investigative competences for university professors]. Laurus, vol. 15, núm. 30, mayo-agosto, pp. 118-137. Universidad Pedagógica Experimental Libertador. Caracas, Venezuela. Recuperado de: https://n9.cl/o87r3

Poveda, J., Chirino, M. (2015). El desarrollo de habilidades investigativas en estudiantes de derecho. Una necesidad social y académica. [The development of investigative skills in law students. A social and academic need]. VARONA, núm. 61, julio-diciembre, pp. 1-10. Universidad Pedagógica Enrique José Varona. La Habana, Cuba. Recuperado de: https://n9.cl/g0qln

Ríos Muñoz, Daniel. (2004). Rasgos de personalidad de profesores innovadores: autonomía, persistencia y orden. [Personality traits of innovative teachers: autonomy, persistence and order]. Revista Latinoamericana de Estudios Educativos (México), vol. XXXIV, núm. 2, 2o trimestre, pp. 95-112. Centro de Estudios Educativos, A.C. Distrito Federal, México. Recuperado de: https://n9.cl/smajh

Reiban, R. (2018). Las competencias investigativas del docente universitario. [The research competences of the University Professor]. Universidad y Sociedad vol.10 no.4 Cienfuegos jul.-set. Recuperado de: https://n9.cl/exc9h 


\section{CIENCIAMATRIA}

Revista Interdisciplinaria de Humanidades, Educación, Ciencia y Tecnología

Año VIII. Vol. VIII. Nro 1. Edición Especial. 2022

Hecho el depósito de ley: pp201602FA4721

ISSN-L: 2542-3029; ISSN: 2610-802X

Universidad Nacional Experimental Francisco de Miranda (UNEFM). Santa Ana de Coro. Venezuela

Alexandra-del-Rocío Maldonado-Núñez; Zila Isabel Esteves-Fajardo

Rivas, L. (2011). Las nueve competencias de un investigador. [The nine competencies of a researcher]. Investigación Administrativa, núm. 108, julio-diciembre, pp. 34-54. Escuela Superior de Comercio y Administración, Unidad Santo Tomás. Distrito Federal, México. Recuperado de: https://n9.cl/fgy3d

Tornimbeni, S. González, C., Corigliani, S. y Co. (2011). Concepciones de expertos sobre las competencias para investigar en Psicología. [Experts' conceptions about the competences to research in Psychology]. Enseñanza e Investigación en Psicología, vol. 16, núm. 1, enero-junio, pp. 5-13. Consejo Nacional para la Enseñanza en Investigación en Psicología A.C. Xalapa, México. Recuperado de: https://n9.cl/ivmrl

Villalobos, J., de Cabrera, Carmen M. (2009). Los docentes y su necesidad de ejercer una práctica reflexiva. [Teachers and their need to exercise reflective practice]. Revista de Teoría y Didáctica de las Ciencias Sociales, núm. 14, enero-junio, pp. 139-166. Universidad de los Andes Mérida, Venezuela. Recuperado de: https://n9.cl/1xqp4

C2022 por los autores. Este artículo es de acceso abierto y distribuido según los términos y condiciones de la licencia Creative Commons Atribución-NoComercial-Compartirlgual 4.0 Internacional (CC BY-NC-SA 4.0)

(https://creativecommons.org/licenses/by-nc-sa/4.0/). 